\title{
Complex Dynamics of Beddington-DeAngelis-Type Predator-Prey Model with Nonlinear Impulsive Control
}

\author{
Changtong Li $(\mathbb{D}$, Xiaozhou Feng $(\mathbb{D}$, Yuzhen Wang $\mathbb{D}$, and Xiaomin Wang $(\mathbb{D}$ \\ School of Science, Xi'an Technological University, Xi'an 710032, China \\ Correspondence should be addressed to Xiaozhou Feng; flxzfxz8@163.com
}

Received 28 August 2020; Revised 28 October 2020; Accepted 18 November 2020; Published 11 December 2020

Academic Editor: Xinzhu Meng

Copyright (C) 2020 Changtong Li et al. This is an open access article distributed under the Creative Commons Attribution License, which permits unrestricted use, distribution, and reproduction in any medium, provided the original work is properly cited.

\begin{abstract}
According to resource limitation, a more realistic pest management is that the impulsive control actions should be adjusted according to the densities of both pest and natural enemy in the field, which result in nonlinear impulsive control. Therefore, we have proposed a Beddington-DeAngelis interference predator-prey model concerning integrated pest management with both density-dependent pest and natural enemy population. We find that the pest-eradication periodic solution is globally stable if the impulsive period is less than the critical value by Floquet theorem. The condition of permanent is established, and a stable positive periodic solution appears via a supercritical bifurcation by bifurcation theorem. Finally, in order to investigate the effects of those nonlinear control strategies on the successful pest control, the bifurcation diagrams showed that the model exists with very complex dynamics. Consequently, the resource limitation may result in pest outbreak in complex ways, which means that the pest control strategies should be carefully designed.
\end{abstract}

\section{Introduction}

Since pest outbreak can cause serious economic loss, pest control has been becoming an increasing concern to entomologists and society all over the world. Several pest control strategies can be used for farmers. As well known, chemical pesticide can directly and rapidly kill large proportion of pest, and it is the only way to prevent economic losses in many cases. Biological control is the practice of releasing of natural enemies to control pests $[1,2]$, sometimes which has a highly efficacious and more active role in some pest situations. However, in order to avoid the resistance development of pests to the control tactic and to protect the environment quality, different pest control techniques should be combined together rather than against overuse of a single control strategy. In particular, integrated pest management(IPM) incorporates a variety of cultural, biological, and chemical methods to high efficiency control of the pest populations, which has been proved that it is more efficient long-term strategy for pest control than the classical one (such as biological control or chemical control) [3-5].
It is reasonable and accurate that impulsive differential equations mathematically simulate the evolution of biological behaviors and complex biological phenomena, which provide conditions for people to assist in the design of IPM strategies and understand the biological phenomena from a mathematical point of view $[1,3,6-9]$. In recent years, the impulsive differential systems with integrated pest management have been systematically studied and developed rapidly $[10,11]$, which enriched its basic theory and analytical techniques of impulsive differential system [12-20]. However, one of the main assumptions in previous literature is that a certain proportion of pest population is killed when the pesticide is applied. Meanwhile, a constant natural enemy is released [21-28], which means that the agricultural resources have almost no effect on IPM.

In reality, the release methods and ratios of numbers of natural enemies will inevitably be affected by the limitation of agricultural resources because of the unbalance development of agricultural, such as agricultural capital, labour forces, biological resources, and pesticides. Therefore, the release ratios of numbers of natural enemies according to current density in the field could significantly affect the effect 
of pest control strategy. In order to take the resource limitation into the IPM strategy, several predator-prey models with nonlinear impulse have been proposed [29-32] and mainly focused on establishing the global stability conditions. However, the nonlinear impulsive function mentioned above is only related to the density of the natural enemy population in the field. Based on resource limitation, the densities of the pest and natural enemy should be carefully monitored before IPM measures are applied. A more realistic case is that the methods for the instantaneous releasing numbers of natural enemies should be based on the dynamic changes of pest and natural enemy densities. In other words, the higher the number of pest population or the lower the number of predator population in the field, the higher the number of predator population should be released and vice versa, which has not been studied until now.

Therefore, in order to take the resource limitation into account and to understand how the nonlinear density regulatory factor for the natural enemies affect the dynamics of predator-prey model, we propose the following predatorprey model with Beddington-DeAngelis functional response and nonlinear impulsive control:

$$
\left\{\begin{array}{l}
\frac{\mathrm{d} x(t)}{\mathrm{d} t}=r x(t)\left(1-\frac{x(t)}{K}\right)-\frac{\alpha x(t) y(t)}{c+x(t)+b y(t)} \\
\frac{\mathrm{d} y(t)}{\mathrm{d} t}=\frac{\beta x(t) y(t)}{c+x(t)+b y(t)}-\delta y(t) \\
x\left(t^{+}\right)=q_{1} x(t) \\
y\left(t^{+}\right)=q_{2} y(t)+\frac{\lambda_{1} x(t)}{1+\theta_{1} x(t)}+\frac{\lambda_{2}}{1+\theta_{2} y(t)}
\end{array}\right\}, \quad t \neq n T,
$$

where $x(t)$ and $y(t)$ are the densities of prey and predator populations, respectively, and all parameters are positive constants. IPM strategy (the nonlinear impulse) is applied at each discrete time point $n T .0 \leq q_{1}, q_{2} \leq 1$ present survival rate of prey and predator after harvesting or pesticides; $q_{2} \geq 1$ means that the pesticides only affect the pest and an impulsive increase of the predator population density is induced by release of predators. Moreover, we choose the nonlinear saturation functions or density-dependent functions as follows:

$$
y\left(t^{+}\right)=q_{2} y(t)+\frac{\lambda_{1} x(t)}{1+\theta_{1} x(t)}+\frac{\lambda_{2}}{1+\theta_{2} y(t)}, \quad t=n T,
$$

$\lambda_{1}, \lambda_{2} \geq 0$ is the maximal release amount of the predator according to the densities of prey and predator populations respectively, and $\theta_{1}, \theta_{2} \geq 0$ represent the shape parameter. In particular, the system with $\lambda_{1}=0, \theta_{2}=0$ (i.e., linear impulsive perturbations) has been investigated in $[27,28]$. We assume that the densities of the natural enemy populations are updated to $y\left(t^{+}\right)=q_{2} y(t)+\left(\lambda_{1} x(t) / 1+\theta_{1} x(t)\right)$ $+\left(\lambda_{2} / 1+\theta_{2} y(t)\right)$ at each time point $n T$, which is a more reasonable control strategy than previous literature [31,32].

The purpose of this paper proposes a Beddington-DeAngelis interference model with nonlinear impulsive control to address how the nonlinear impulsive control actions affect the successful pest control strategies. By using the Floquet theorem and small-amplitude perturbation skills, we obtain that the pest-eradication periodic solution is globally stable if the period of impulsive $T$ is less than a critical value, and a sufficient condition for the permanence of the system is obtained. Moreover, when the trivial periodic solution loses its stability, we obtain that a nontrivial periodic solution appears via a supercritical bifurcation by employing a bifurcation theorem. By bifurcation diagrams, we show that the model presents more rich and interesting dynamic behavior including periodic doubling bifurcation, period-halving bifurcations, chaotic solutions, and multistability. Finally, we give some related biological implications.

\section{Global Stability of the Pest-Eradication Periodic Solution}

As we know, eradicating the pest population is an important purpose of IPM strategy, so the existence and global stability of the pest-eradication periodic solution play a crucial role in studying the dynamical behavior. For this, we firstly study the properties of the subsystem

$$
\begin{cases}\frac{\mathrm{d} y(t)}{\mathrm{d} t}=-\delta y(t), & t \neq n T, \\ y\left(t^{+}\right)=q_{2} y(t)+\frac{\lambda_{2}}{1+\theta_{2} y(t)}, & t=n T .\end{cases}
$$

Subsystem (3) is a nonlinear growth model, by using the same methods as those in reference [32], and we can have the following result.

Lemma 1. When $1-q_{2} \exp (-\delta T)>0$, model (3) has a globally stable periodic solution:

$$
y_{p}(t) y^{*} \exp (-\delta(t-n T)), \quad t \in(n T,(n+1) T),
$$

where $y^{*}=\left((\sqrt{A+1}-1) /\left(2 \theta_{2} \exp (-\delta T)\right)\right) \quad$ with $A=\left(\left(4 \lambda_{2} \theta_{2} \exp (-\delta T)\right) /\left(1-q_{2} \exp (-\delta T)\right)\right)$ is a positive constant.

Therefore, when $q_{2} \exp (-\delta T)<1$, we have that model (1) has complete expression for pest-eradication periodic solution $\left(x_{p}(t), y_{p}(t)\right)=\left(0, y^{*} \exp (-\delta(t-n T))\right)$.

Next, we will present a condition which guarantees the local and global asymptotic stability of pest-eradication periodic solution $\left(x_{p}(t), y_{p}(t)\right)$ of model (1).

Theorem 1. The pest-eradication periodic solution $\left(x_{p}(t), y_{p}(t)\right)$ is locally asymptotically stable provided that

$$
T<\frac{1}{r} \ln \frac{1}{q_{1}}-\frac{\alpha}{r b \delta} \ln \left(\frac{c+b y^{*} \exp (-\delta T)}{c+b y^{*}}\right) .
$$


Furthermore, $\left(x_{p}(t), y_{p}(t)\right)$ is globally asymptotically stable provided

$$
T<\frac{1}{r} \ln \frac{1}{q_{1}}-\frac{\alpha}{r b \delta} \ln \left(\frac{c+K+b y^{*} \exp (-\delta T)}{c+K+b y^{*}}\right) .
$$

Proof. The local stability of the pest-eradication solution may be determined by the behavior of small-amplitude perturbations of the solution. Defining $u(t)=x(t)-$ $x_{p}(t), v(t)=y(t)-y_{p}(t)$, then the fundamental matrix $\Phi(t)$ of model (1) satisfies

$$
\frac{\mathrm{d} \Phi(t)}{\mathrm{d} t}=\left(\begin{array}{cc}
r-\frac{\alpha y_{p}(t)}{c+b y_{p}(t)} & 0 \\
\frac{\beta y_{p}(t)}{c+b y_{p}(t)} & -\delta
\end{array}\right) \Phi(t)
$$

$$
\begin{aligned}
M & =B(T) \Phi(T) \\
& =\left(\begin{array}{c}
q_{1} \exp \left(\int_{0}^{T}\left(r-\frac{\alpha y_{p}(t)}{c+b y_{p}(t)}\right) \mathrm{d} t\right) \\
*
\end{array}\right.
\end{aligned}
$$

has absolute value less than one, then the solution $\left(x_{p}(t), y_{p}(t)\right)$ of model (1) is locally stable, and $*$ is not needed to calculate the exact form. Note that all multipliers are

$$
\begin{aligned}
& \mu_{1}=q_{1} \exp \left(\int_{0}^{T}\left(r-\frac{\alpha y_{p}(t)}{c+b y_{p}(t)}\right) \mathrm{d} t\right), \\
& \mu_{2}=\left(q_{2}-\frac{\lambda_{2} \theta_{2}}{\left(1+\theta_{2} y_{p}(T)\right)^{2}}\right) \exp (-\delta T) .
\end{aligned}
$$

It is easy to see that $\mu_{2} \leq q_{2} \exp (-\delta T)<1$. Furthermore,

$$
\frac{\lambda_{2} \theta_{2} \exp (-\delta T)}{\left(1+\theta_{2} y_{p}(t)\right)^{2}} \leq \frac{4 \lambda_{2} \theta_{2} \exp (-\delta T)}{\left(\sqrt{1+4 \lambda_{2} \theta_{2} \exp (-\delta T)}+1\right)^{2}}<1 .
$$

So, we obtain $\mu_{2} \geq q_{2} \exp (-\delta T)-1>-1$, which means $\left|\mu_{2}\right|<1$. Since $\int_{0}^{T}\left(r-\left(\alpha y_{p}(t) / c+b y_{p}(t)\right)\right) \mathrm{d} t=(1 / b \delta)$, ln $\left(\left(c+b y^{*} \exp (-\delta T)\right) /\left(c+b y^{*}\right)\right)$, according to the Floquet theory of impulsive differential equation, the pest-eradication periodic solution $\left(x_{p}(t), y_{p}(t)\right)$ is locally asymptotically stable if

$$
T<\frac{1}{r} \ln \frac{1}{q_{1}}-\frac{\alpha}{r b \delta} \ln \left(\frac{c+b y^{*} \exp (-\delta T)}{c+b y^{*}}\right) .
$$

and $\Phi(0)=I$ is the identity matrix. From the third and fourth equations of (1), one has that

$$
\begin{aligned}
\left(\begin{array}{c}
u\left(n T^{+}\right) \\
v\left(n T^{+}\right)
\end{array}\right) & =\left(\begin{array}{cc}
q_{1} & 0 \\
\lambda_{1} & q_{2}-\frac{\lambda_{2} \theta_{2}}{\left(1+\theta_{2} y_{p}(T)\right)^{2}}
\end{array}\right)\left(\begin{array}{c}
u(n T) \\
v(n T)
\end{array}\right) \\
& =B(T)\left(\begin{array}{l}
u(n T) \\
v(n T)
\end{array}\right) .
\end{aligned}
$$

Therefore, if each eigenvalues of the following matrix

$$
\left.\left(q_{2}-\frac{\lambda_{2} \theta_{2}}{\left(1+\theta_{2} y_{p}(T)\right)^{2}}\right) \exp (-\delta T)\right)
$$

Next, we will show the global attractivity provided condition (6) is satisfied. From the comparison theorem of impulsive equation, we obtain $y(t) \geq y_{p}(t)-\varepsilon$ for all $t$ large enough. Also, it is easy to see that $x(t)<K+\varepsilon$ for all $t$ large enough.

For simplicity, we may assume that $y(t) \geq y_{p}(t)-\varepsilon$ and $x(t)<K+\varepsilon$ for all $t \geq 0$. If condition (6) holds true, then we choose an $\varepsilon>0$ such that

$$
\eta \triangleq q_{1} \exp \left(\int_{0}^{T}\left(r-\frac{\alpha\left(y_{p}(t)-\varepsilon\right)}{c+K+\varepsilon+b\left(y_{p}(t)-\varepsilon\right)}\right) \mathrm{d} t\right)<1 .
$$

From model (1), we obtain

$$
\begin{cases}\frac{\mathrm{d} x(t)}{\mathrm{d} t} \leq x(t)\left(r-\frac{\alpha\left(y_{p}(t)-\varepsilon\right)}{c+K+\varepsilon+b\left(y_{p}(t)-\varepsilon\right)}\right), & t \neq n T, \\ x\left(t^{+}\right)=q_{1} x(t), & t=n T .\end{cases}
$$

Integrating on $(n T,(n+1) T]$, one obtains 


$$
\begin{aligned}
x((n+1) T) & =q_{1} x(n T) \exp \left[\int_{n T}^{(n+1) T}\left(r-\frac{\alpha\left(y_{p}(t)-\varepsilon\right)}{c+K+\varepsilon+b\left(y_{p}(t)-\varepsilon\right)}\right) \mathrm{d} t\right] \\
& =x(n T) \eta .
\end{aligned}
$$

Thus, $x(n T)=x\left(0^{+}\right) \eta^{n}$ and consequently $x(n T) \longrightarrow 0$ as $n \longrightarrow \infty$. Therefore, $x(t) \longrightarrow 0$ as $n \longrightarrow \infty$, since $0<x(t) \leq q_{1} x(n T) \exp (r T)$ for $t \in(n T,(n+1) T]$.

Following, we only need to prove $y(t) \longrightarrow y_{p}(t)$ as $t \longrightarrow \infty$. There must exist a $0<\varepsilon_{1}<(\delta c / \beta)$ and $T_{1}>0$ such that $0<x(t)<\varepsilon_{1}$ for $t>T_{1}$. Again, for simplicity, it is assumed that $0<x(t)<\varepsilon_{1}$ holds true for $t \geq 0$. Then, we deduce that $\left(\lambda_{1} x(t) / 1+\theta_{1} x(t)\right)<\varepsilon_{1} \lambda_{1}$ and

$$
-\delta y(t) \leq \frac{\mathrm{d} y}{\mathrm{~d} t} \leq y(t)\left(\frac{\beta \varepsilon_{1}}{c}-\delta\right),
$$

from which we can have the following equation:

$$
\begin{cases}\frac{\mathrm{d} z(t)}{\mathrm{d} t}=\left(\frac{\beta \varepsilon_{1}}{c}-\delta\right) z(t)=-\delta_{1} z(t), & t \neq n T, \\ z\left(t^{+}\right)=q_{2} z(t)+\varepsilon_{1} \lambda_{1}+\frac{\lambda_{2}}{1+\theta_{2} z(t)}, \quad t=n T .\end{cases}
$$

By Lemma 1, model (17) has a globally asymptotically stable periodic solution $z_{p}(t)=z^{*} \exp \left(-\delta_{1}(t-n T)\right)$, where $z^{*}=\left(\left(-A_{1}+\sqrt{A_{1}^{2}+4 B_{1}\left(\varepsilon_{1} \lambda_{1}+\lambda_{2}\right)}\right) /\left(2 B_{1}\right)\right)$ with $A_{1}=1-$ $\left(q_{2}+\varepsilon_{1} \lambda_{1} \theta_{2}\right) \exp \left(-\delta_{1} T\right), B_{1}=\theta_{2}(1-$

$\left.q_{2} \exp \left(-\delta_{1} T\right)\right) \exp \left(-\delta_{1} T\right)$ and $t \in(n T,(n+1) T]$.

According to the comparison theorem, we get $y_{p}(t) \leq y(t) \leq z(t)$ and $z(t) \longrightarrow z_{p}(t), z_{p}(t) \longrightarrow y_{p}(t)$ as $t \longrightarrow \infty$. Hence, for any $\varepsilon_{1}>0$, we have

$$
y_{p}(t)-\varepsilon_{1}<y(t)<z_{p}(t)+\varepsilon_{1},
$$

for $T_{2} \geq T_{1}>0$. Furthermore, let $\varepsilon \longrightarrow 0$, we get $y_{p}(t)-$ $\varepsilon_{1}<y(t)<y_{p}(t)+\varepsilon_{1}$ for $t$ large enough. In other words, $y(t) \longrightarrow y_{p}(t)$ as $t \longrightarrow \infty$ for $t$ large enough. The proof is completed.

\section{Permanence}

Persistence is an important property of dynamical systems for addressing the long-term survival of all components of a system. Now, we investigate the sufficient condition for the permanence of model (1).

Theorem 2. Model (1) is permanent if $T>(1 / r) \ln \left(1 / q_{1}\right)-$ $(\alpha / r b \delta) \ln \left(c+b y^{*} \exp (-\delta T) / c+b y^{*}\right)$ holds true.

Proof. Suppose that $(x(t), y(t))$ is a solution of (1) with $x(0)>0, y(0)>0$. It is easy to know that $x(t)<M, y(t)<M$ for all $t>0, M>(r c / \alpha)$. Define $m_{2}=y^{*} \exp (-\delta T)-\varepsilon$. From Theorem 1 , it is easy to see that $y(t) \geq m_{2}$ for $t$ large enough.
Then, we shall find an $m_{1}$ such that $x(t) \geq m_{1}$ for all $t$ that are large enough. We will do it in the following two steps.

Step 1. Let $m_{3}>0, \varepsilon_{1}>0$ be small enough such that $\delta_{2}=$ $\delta-\left(\beta m_{3} / c+m_{3}\right)>0$ and $\eta \triangleq q_{1} \exp \left(r T-\left(r m_{3} T / K\right)-\right.$ $\left.\left(\alpha / b \delta_{2}\right) \ln \left(c+b u^{*} \exp \left(-\delta_{2} T\right) / c+b \quad u^{*}\right)-\left(\alpha \varepsilon_{1} T / c\right)\right)>1$, where $u^{*}=\left(\left(-A_{2}+\sqrt{A_{2}^{2}+4 B_{2}\left(m_{3} \lambda_{1}+\lambda_{2}\right)}\right) /\left(2 B_{2}\right)\right)$ with $\quad A_{2}=1-\left(q_{2}+m_{3} \lambda_{1} \theta_{2}\right) \exp \left(-\delta_{2} T\right), B_{2}=\theta_{2}\left(1-q_{2}\right.$ $\left.\exp \left(-\delta_{2} T\right)\right) \exp \left(-\delta_{2} T\right)$. We will prove that $x(t)<m_{3}$ cannot hold for all $t>0$. Otherwise,

$$
\frac{\mathrm{d} y(t)}{\mathrm{d} t} \leq-\delta_{2} y(t) \text {. }
$$

From Lemma 1, we then obtain $y(t) \leq u(t)$ and $u(t) \longrightarrow u_{p}(t), t \longrightarrow \infty$, where $u(t)$ is the solution of

$\begin{cases}\frac{\mathrm{d} u(t)}{\mathrm{d} t}=-\delta_{2} u(t), & t \neq n T, \\ u\left(t^{+}\right) \leq q_{2} u(t)+\lambda_{1} m_{3}+\frac{\lambda_{2}}{1+\theta_{2} u(t)}, & t=n T,\end{cases}$

and $u_{p}(t)=u^{*} \exp \left(-\delta_{2}(t-n T)\right), t \in(n T,(n+1) T]$ with $u^{*}=\left(\left(-A_{2}+\sqrt{A_{2}^{2}+4 B_{2}\left(m_{3} \lambda_{1}+\lambda_{2}\right)}\right) /\left(2 B_{2}\right)\right)$.

Consequently, there exists a $\widehat{T}>0$ such that $y(t) \leq u(t)<u_{p}(t)+\varepsilon_{1}$ and

$$
\begin{aligned}
\frac{\mathrm{d} x(t)}{\mathrm{d} t} & \left.\geq x(t)\left(r-\frac{r m_{3}}{K}-\frac{\alpha y(t)}{c+b y(t)}\right)\right) \\
& \left.\geq x(t)\left(r-\frac{r m_{3}}{K}-\frac{\alpha u_{p}(t)+\alpha \varepsilon_{1}}{c+b u_{p}(t)+b \varepsilon_{1}}\right)\right) \\
& \left.\geq x(t)\left(r-\frac{r m_{3}}{K}-\frac{\alpha u_{p}(t)}{c+b u_{p}(t)}-\frac{\alpha \varepsilon_{1}}{c}\right)\right),
\end{aligned}
$$

for $t>\widehat{T}$. Furthermore, we get

$$
\begin{cases}\left.\frac{\mathrm{d} x(t)}{\mathrm{d} t} \geq x(t)\left(r-\frac{r m_{3}}{K}-\frac{\alpha u_{p}(t)}{c+b u_{p}(t)}-\frac{\alpha \varepsilon_{1}}{c}\right)\right), & t \neq n T, \\ x\left(t^{+}\right)=q_{1} x(t), & t=n T,\end{cases}
$$

for $t>\widehat{T}$. Integrating (22) on $(n T,(n+1) T]$, where $n T>\widehat{T}$, we obtain

$$
\begin{aligned}
x((n+1) T) & \geq x\left(n T^{+}\right) \exp \left(\int_{n T}^{(n+1) T}\left(r-\frac{r m_{3}}{K}-\frac{\alpha u_{p}(t)}{c+b u_{p}(t)}-\frac{\alpha \varepsilon_{1}}{c}\right) \mathrm{d} t\right) \\
& =x(n T) \eta .
\end{aligned}
$$


Then, $\quad x((n+k) T)>x(n T) \eta^{n} \longrightarrow \infty$ as $k \longrightarrow \infty$, which is a contradiction. Therefore, there exists a $t_{1}>0$ such that $x\left(t_{1}\right) \geq m_{3}$.

Step 2. If $x(t) \geq m_{3}$ for all $t \geq t_{1}$, then model (1) is permanent. If not, we can define $t^{*}=\inf _{t>t_{1}}\left\{x(t)<m_{3}\right\}$, Then, $x(t) \geq m_{3}$ for $t \in\left[t_{1}, t^{*}\right]$ since the continuity of $x(t)$ and $x\left(t^{*}\right)=m_{3}$. We only need to consider two possible cases.

Case (1): $t^{*}=n_{1} T$. For some $n_{1} \in Z_{+}$, then we have $q_{1} m_{3} \leq x\left(t^{*+}\right)=q_{1} x\left(t^{*}\right)<m_{3}$. Select $n_{2}, n_{3} \in Z_{+}$such that

$$
q_{1}^{n_{2}} \eta^{n_{3}} \exp \left(\left(n_{2}+1\right) \eta_{1} T\right)>1,
$$

where $\quad \rho=r-\left(m_{3} r / K\right)-(\alpha / c) M<0 . \quad$ Let $T^{\prime}=n_{2} T+n_{3} T$, we will show that there exists a $t^{*}<t_{2} \leq t^{*}+T^{\prime}$ such that $x\left(t_{2}\right) \geq m_{3}$. If not, by (20) with $u\left(t^{*+}\right)=y\left(t^{*+}\right)$, we can see that $y(t) \leq u(t) \leq u_{p}(t)+\varepsilon_{1}$ for $t \in\left[t^{*}+n_{2} T, t^{*}+T^{\prime}\right]$. An argument similar to Step 1 yields

$$
x\left(t^{*}+T^{\prime}\right) \geq x\left(t^{*}+n_{2} T\right) \eta^{n_{3}}
$$

Since $y(t) \leq M$, when $t \in\left[t^{*}, t^{*}+n_{2} T\right]$, we get

$$
\begin{cases}\frac{\mathrm{d} x(t)}{\mathrm{d} t} \geq x(t)\left(r-\frac{m_{3} r}{K}-\frac{\alpha}{c} M\right), & t \neq n T, \\ x\left(t^{+}\right)=q_{1} x(t), & t=n T .\end{cases}
$$

Furthermore, we integrate equation (26) on $\left[t^{*}, t^{*}+n_{2} T\right]$, then we know

$$
x\left(t^{*}+n_{2} T\right) \geq m_{3} q_{1}^{n_{2}-1} \exp \left(n_{2} \rho T\right) .
$$

From above, we obtain $x\left(t^{*}+T^{\prime}\right) \geq m_{3} q_{1}^{n_{2}-1}$ $\exp \left(n_{2} \rho T\right) \eta^{n_{3}}>m_{3}$ which leads to a contradiction.

Now, let $\tilde{t}=\inf _{t>t^{*}}\left\{x(t) \geq m_{3}\right\}$, then $x(t) \leq m_{3}$ for $t \in\left(t^{*}, \widetilde{t}\right)$ and $x(\widetilde{t})=m_{3}$. For any $t^{*}<t<\widetilde{t}$, let us assume that there exists $k \in Z_{+}$such that $t \in\left(t^{*}+(k-\right.$ 1) $\left.T, t^{*}+k T\right]$ and $k \leq n_{2}+n_{3}$, so, from (26), we obtain

$$
\begin{aligned}
x(t) & \geq x\left(t^{*+}\right) q_{1}^{k-1} \exp ((k-1) \rho T) \exp \left(\rho\left(t-\left(t^{*}+(k-1) T\right)\right)\right) \\
& \geq m_{3} q_{1}^{k} \exp (k \rho T) \\
& \geq m_{3} q_{1}^{n_{2}+n_{3}} \exp \left(\left(n_{2}+n_{3}\right) \rho T\right) .
\end{aligned}
$$

Let $\quad m_{1}^{\prime}=m_{3} q_{1}^{n_{2}+n_{3}} \exp \left(\left(n_{2}+n_{3}\right) \eta_{1} T\right)$, thus, for $t \in\left(t^{*}, \tilde{t}\right)$, we get $x(t) \geq m_{1}^{\prime}$. For $t>\widetilde{t}$, we can continue the same arguments since $x(\widetilde{t}) \geq m_{3}$.

Case (2): $t^{*} \neq n_{1} T, n_{1} \in Z_{+}$. We have $x\left(t^{*}\right)=m_{3}$ since $x(t)$ is continuous. Suppose $t^{*} \in\left(n_{1}^{\prime} T,\left(n_{1}^{\prime}+1\right) T\right), n_{1}^{\prime} \in Z_{+}$, we consider the following two cases for $t \in\left(t^{*},\left(n_{1}^{\prime}+1\right) T\right)$.

Case (2a): $x(t)<m_{3}$ for $t \in\left(t^{*},\left(n_{1}^{\prime}+1\right) T\right]$. In this case, we continue this process by using step case (1), we can prove that there exists a $\hat{t}=\inf _{t>t^{*}}\left\{x(t) \geq m_{3}\right\}$ such that $x(t)<m_{3}, t \in\left(t^{*}, \widehat{t}\right)$, and $x(\hat{t})=m_{3}$. For any $t \in\left(t^{*}, \widehat{t}\right)$ suppose $t \in\left(n_{1}^{\prime} T+\left(l^{\prime}-1\right) T, n_{1}^{\prime} T+l^{\prime} T\right], l^{\prime} \leq 1+n_{2}+n_{3}$, we obtain

$$
\begin{aligned}
x(t) & \geq m_{3} q_{1}^{l^{\prime}-1} \exp \left(l^{\prime} \eta_{1} T\right) \\
& \geq m_{3} q_{1}^{n_{2}+n_{3}} \exp \left(\left(n_{2}+n_{3}+1\right) \rho T\right) \triangleq m_{1} .
\end{aligned}
$$

Since $m_{1}<m_{1}^{\prime}$, thus, for $t \in\left(t^{*}, \widehat{t}\right)$, we have $x(t) \geq m_{1}$. Case (2b): there is a $t^{\prime} \in\left(t^{*},\left(n_{1}^{\prime}+1\right) T\right)$ such that $x\left(t^{\prime}\right) \geq m_{3}$. In this case, a similar argument as above, there is $\bar{t}=\inf _{t>t^{*}}\left\{x(t)>m_{3}\right\} \quad$ such that $x(t)<m_{3}, t \in\left(t^{*}, \bar{t}\right)$. Therefore, integrating equation (26) on $\left.\left[t^{*}, t\right)(t \leq \bar{t})\right)$, we can get that $x(t) \geq x\left(t^{*}\right) \exp \left(\rho\left(t-t^{*}\right)\right) \geq m_{1}$. Thus, the similar argument can be continued for both cases since $x(t) \geq m_{1}$ for some $t>t_{1}$. This completes the proof.

Remark 1. Define $\quad T^{*}=(1 / r) \ln \left(1 / q_{1}\right)-(\alpha / r b \delta) \ln$ $\left(\left(c+b y^{*} \exp (-\delta T)\right) /\left(c+b y^{*}\right)\right)$, the pest-eradication periodic solution loses its stability if $T>T^{*}$. Therefore, $T^{*}$ is the critical threshold value to discriminate between stability and permanence.

\section{Bifurcation}

Now, we will deal with the existence of nontrivial solution near the pest-eradication solution. We use the bifurcation theory in earlier publications [33]. 
Theorem 3. Model (1) has a positive nontrivial periodic solution when $T=T^{*}$, which is supercritical if $q_{2}>\theta_{2} \lambda_{2}$ and $K \alpha<4 r b c$.

Proof. It is convenient for the computation to exchange $x$ and $y$ and change the period $T$ to $\tau$.

$$
\left\{\begin{array}{l}
\frac{\mathrm{d} x(t)}{\mathrm{d} t}=\frac{\beta x(t) y(t)}{c+y(t)+b x(t)}-\delta x \\
\frac{\mathrm{d} y(t)}{\mathrm{d} t}=r y\left(1-\frac{y}{K}\right)-\frac{\alpha x(t) y(t)}{c+y(t)+b x(t)}
\end{array}\right\}, \quad t \neq n \tau,
$$

Let $\Phi$ be the solution of the pulse-free system associated with system (30). Also, we get $X(t)=\Phi\left(t, X_{0}\right)$ with $X_{0}=X(0)$. We define the mapping $\Theta_{1}, \Theta_{2}: R^{2} \longrightarrow R^{2}$ by

$$
\begin{aligned}
& \Theta_{1}(x, y)=q_{2} x+\frac{\lambda_{1} y}{1+\theta_{1} y}+\frac{\lambda_{2}}{1+\theta_{2} x}, \\
& \Theta_{2}(x, y)=q_{1} y, \xi(t)=\left(y_{p}(t), 0\right),
\end{aligned}
$$

and the map $F_{1}, F_{2}: R^{2} \longrightarrow R^{2}$ by

$$
\begin{aligned}
& F_{1}(x, y)=\frac{\beta x(t) y(t)}{c+y(t)+b x(t)}-\delta x, \\
& F_{2}(x, y)=\operatorname{ry}\left(1-\frac{y}{K}\right)-\frac{\alpha x(t) y(t)}{c+y(t)+b x(t)} .
\end{aligned}
$$

Furthermore, to establish the nontrivial periodic solution of system (30), we employ the techniques in [33]. Then, we have

$$
\begin{aligned}
& \frac{\partial \Phi_{1}\left(t, X_{0}\right)}{\partial x}=\exp \left(\int_{0}^{t} \frac{\partial F_{1}(\xi(t))}{\partial x} \mathrm{~d} t\right) \\
& \frac{\partial \Phi_{1}\left(t, X_{0}\right)}{\partial y}=\int_{0}^{t} \exp \left(\int_{s}^{t} \frac{\partial F_{1}(\xi(s))}{\partial y} \mathrm{~d} s\right) \frac{\partial F_{1}(\xi(v))}{\partial x} \exp \left(\int_{0}^{v} \frac{\partial F_{2}(\xi(s))}{\partial y} \mathrm{~d} s\right) \mathrm{d} v \\
& \frac{\partial \Phi_{2}\left(t, X_{0}\right)}{\partial x}=0, \\
& \frac{\partial \Phi_{2}\left(t, X_{0}\right)}{\partial y}=\exp \left(\int_{0}^{t} \frac{\partial F_{2}(\xi(t))}{\partial y} \mathrm{~d} t\right)>0 .
\end{aligned}
$$

Thus, by simple calculations, we obtain

$$
\begin{aligned}
d_{0}^{\prime} & =1-\left(\frac{\partial \theta_{2}}{\partial y} \cdot \frac{\partial \Phi_{2}}{\partial y}\right)_{\left(\tau_{0}, X_{0}\right)} \\
& =1-q_{1} \exp \left(\int_{0}^{\tau_{0}}\left(r-\frac{\alpha y_{p}(t)}{c+b y_{p}(t)}\right) \mathrm{d} t\right),
\end{aligned}
$$

where $\tau_{0}$ is the root of $d_{0}=0$. Actually, it is easy to see that $d_{0}^{\prime}=0$ is equivalent to $\tau_{0}=T^{*}$.

Also, we obtain that

$$
\begin{aligned}
a_{0}^{\prime} & =1-\left(\frac{\partial \theta_{1}}{\partial x} \cdot \frac{\partial \Phi_{1}}{\partial x}\right)_{\left(\tau_{0}, X_{0}\right)}=1-q_{1} \exp (-\mathrm{DT})>0, \\
b_{0}^{\prime} & =-\left(\frac{\partial \theta_{1}}{\partial x} \cdot \frac{\partial \Phi_{1}}{\partial y}+\frac{\partial \theta_{1}}{\partial y} \cdot \frac{\partial \Phi_{2}}{\partial y}\right)_{\left(\tau_{0}, X_{0}\right)} \\
& =-\left(q_{2}-\frac{\theta_{2} \lambda_{2}}{\left(1+\theta_{2} y^{*} \exp (-\mathrm{DT})\right)^{2}}\right) \frac{\partial \Phi_{1}\left(\tau_{0}, X_{0}\right)}{\partial y}-\lambda_{1} \frac{\partial \Phi_{2}\left(\tau_{0}, X_{0}\right)}{\partial y} .
\end{aligned}
$$

Note that $b_{0}^{\prime}<0$ if $q_{2}>\theta_{2} \lambda_{2}$.

Following, we should calculate the second-order partial derivatives: 


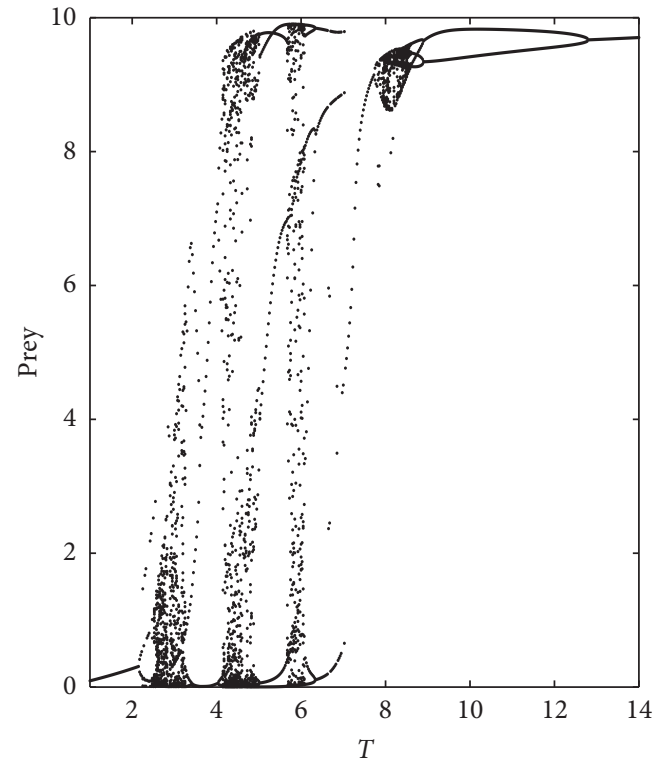

(a)

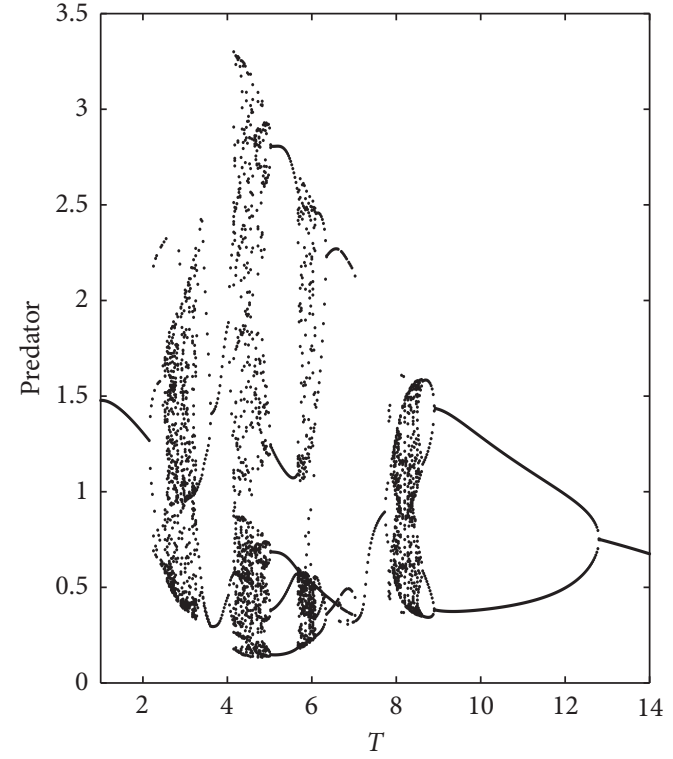

(b)

Figure 1: Bifurcation diagrams of model (1) with respect to $T$. The parameter values are as follows. $r=2.72, K=10, c=1, b=0.001, \alpha=1.2, \beta=0.48, \delta=0.59, \theta_{1}=12, \theta_{2}=1, q_{1}=0.62, q_{2}=0.98, \lambda_{1}=4.4$, and $\lambda_{2}=10.2$.

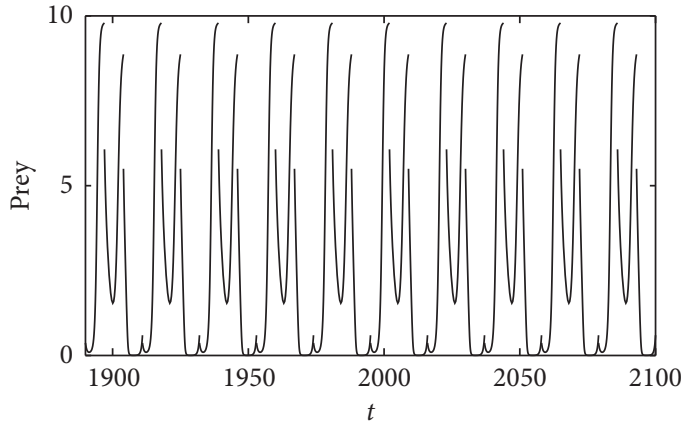

(a)

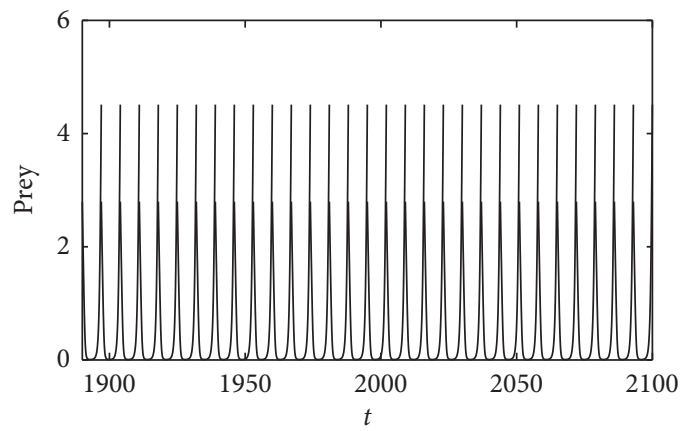

(c)

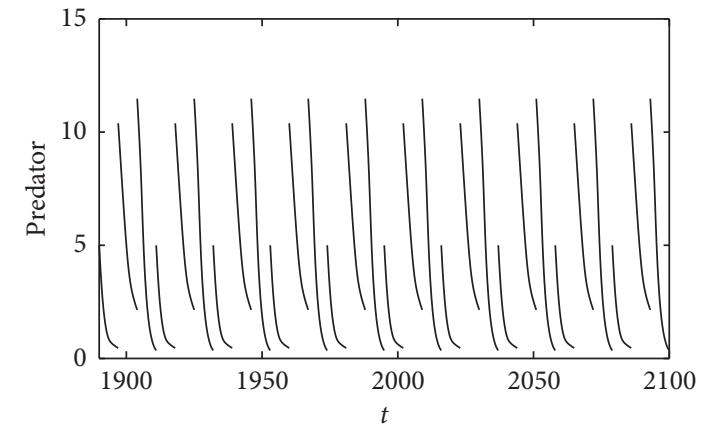

(b)

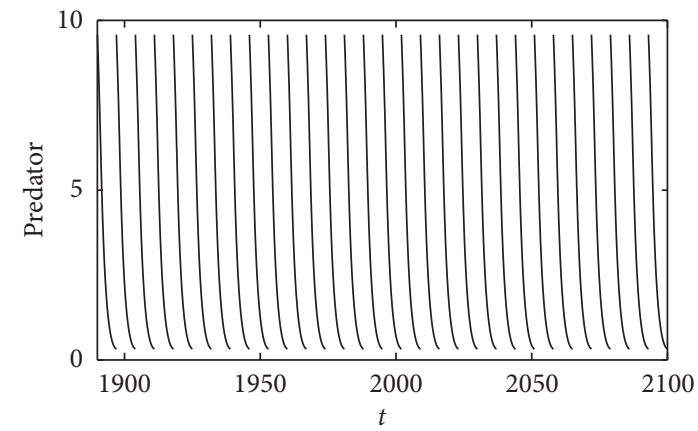

(d)

Figure 2: Two coexisting attractors of model (1) with $T=7$. The other parameter values are identical to those in Figure 1. The initial conditions are as follows: $(A-B)\left(x_{0}, y_{0}\right)=(1.4,2.1) ;(C-D)\left(x_{0}, y_{0}\right)=(1.8,2.6)$. 


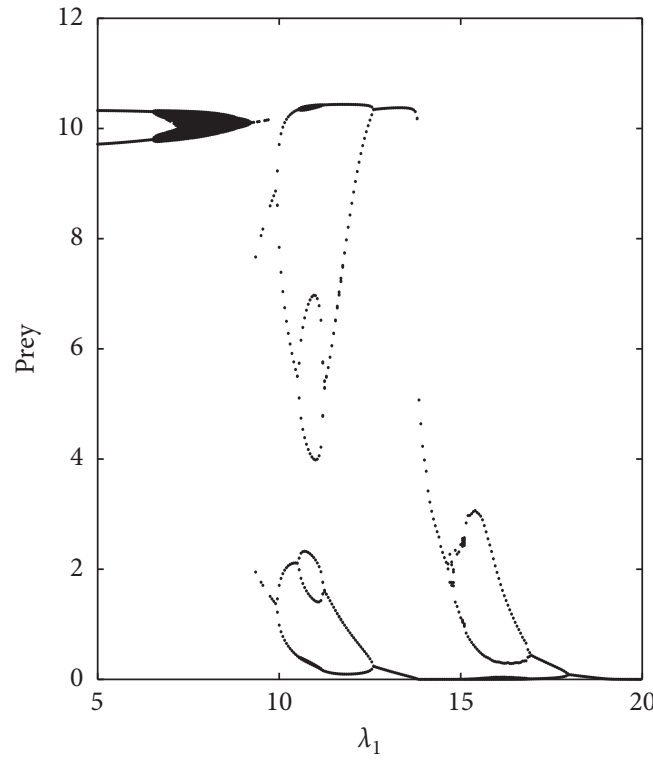

(a)

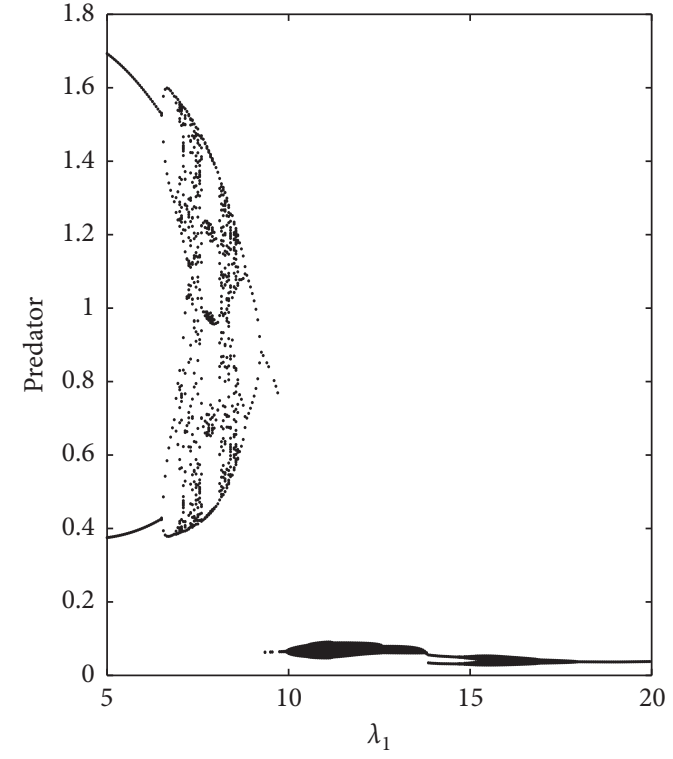

(b)

Figure 3: Bifurcation diagrams of model (1) with respect to $\lambda_{1}$. The other parameters are identical to those in Figure 1 and $K=10.5, T=10$.

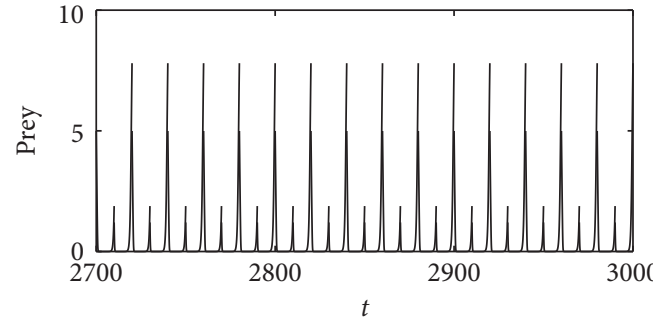

(a)

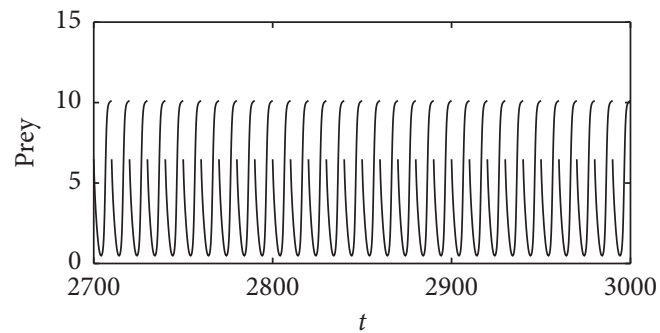

(c)

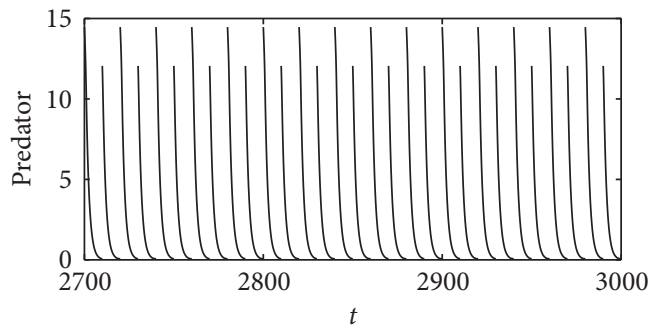

(b)

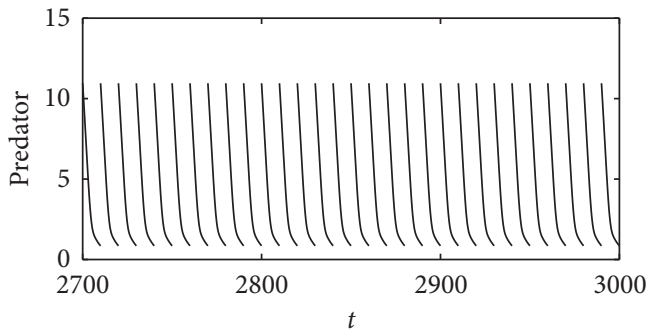

(d)

FIgUre 4: Two coexisting attractors of model (1) with $\lambda_{1}=9.4$. The other parameter values identical to those in Figure 3. The initial conditions are as follows: $(A-B)\left(x_{0}, y_{0}\right)=(1.4,2.1) ;(C-D)\left(x_{0}, y_{0}\right)=(1.8,2.6)$.

$$
\begin{aligned}
\frac{\partial^{2} \Phi_{2}\left(\tau_{0}, X_{0}\right)}{\partial x \partial y} & =\int_{0}^{\tau_{0}} \exp \left(\int_{s}^{\tau_{0}} \frac{\partial F_{2}(\zeta(t))}{\partial y} \mathrm{~d} t\right) \frac{\partial^{2} F_{2}(\zeta(t))}{\partial x \partial y} \exp \left(\int_{0}^{s} \frac{\partial F_{2}(\zeta(t))}{\partial y} \mathrm{~d} t\right) \mathrm{d} s \\
& =-\int_{0}^{\tau_{0}} \exp \left(\int_{s}^{t} \frac{\partial F_{2}(\zeta(t))}{\partial y} \mathrm{~d} t\right) \frac{b \alpha y_{p}(s)}{\left(c+b y_{p}(s)\right)^{3}} \exp \left(\int_{0}^{s} \frac{\partial F_{2}(\zeta(t))}{\partial y} \mathrm{~d} t\right) \mathrm{d} s \\
& <0 .
\end{aligned}
$$




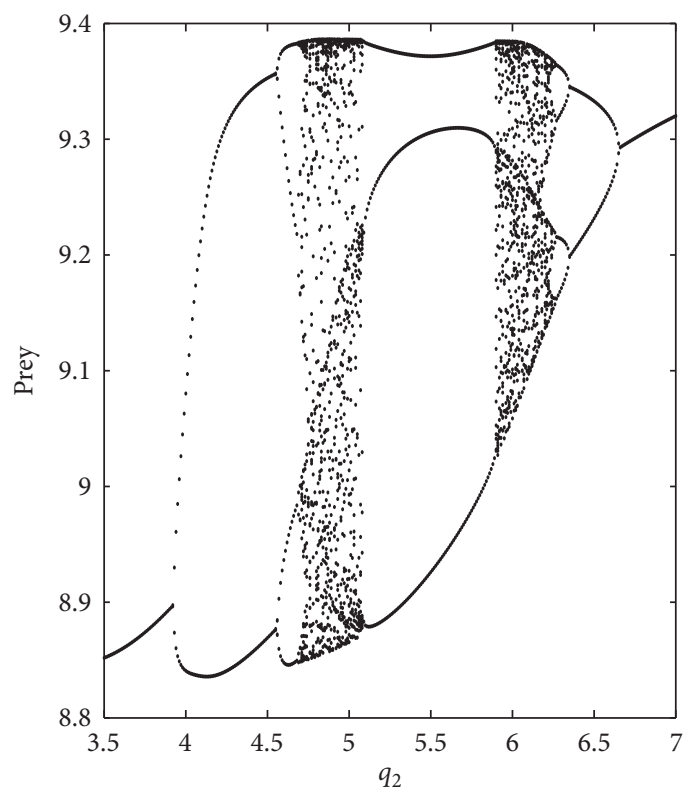

(a)

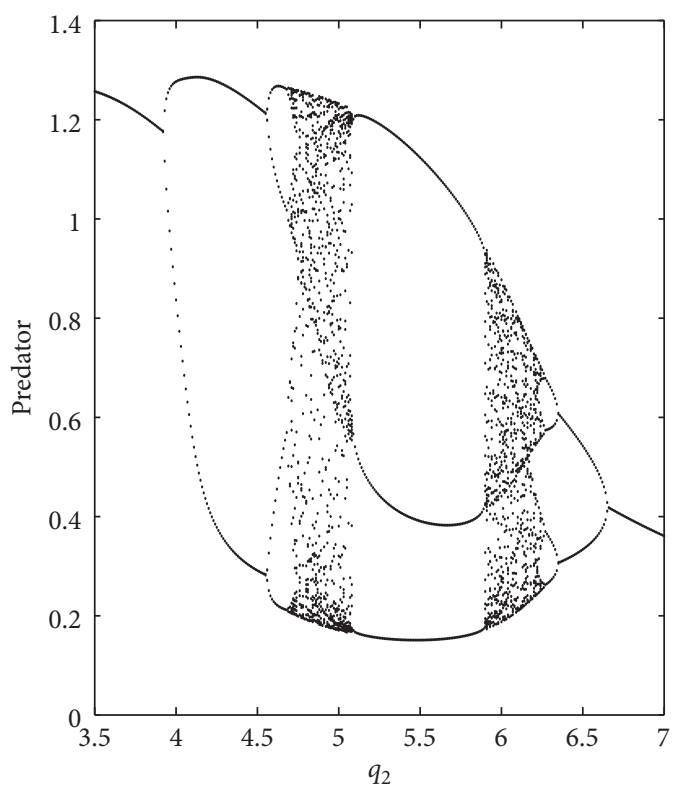

(b)

Figure 5: Bifurcation diagrams of model (1) with respect to $q_{2}$. Parameter values are as follows: $r=2.12, K=10, c=1, b=0.01, \alpha=1.2, \beta=0.48, \delta=0.39, \theta_{1}=12.5, \theta_{2}=1.5, q_{1}=0.11, \lambda_{1}=1.5$, and $\lambda_{2}=2$.

By the same methods as shown above, we have

$$
\begin{aligned}
\frac{\partial^{2} \Phi_{2}\left(\tau_{0}, X_{0}\right)}{\partial y^{2}}= & \int_{0}^{\tau_{0}} \exp \left(\int_{s}^{\tau_{0}} \frac{\partial F_{2}(\zeta(t))}{\partial y} \mathrm{~d} t\right) \frac{\partial^{2} F_{2}(\zeta(t))}{\partial y^{2}} \exp \left(\int_{0}^{s} \frac{\partial F_{2}(\zeta(t))}{\partial y} \mathrm{~d} t\right) \mathrm{d} s \\
& +\int_{0}^{\tau_{0}}\left[\exp \left(\int_{s}^{\tau_{0}} \frac{\partial F_{2}(\zeta(t))}{\partial y} \mathrm{~d} t\right) \frac{\partial^{2} F_{2}(\zeta(s))}{\partial y \partial x} \cdot \int_{0}^{s} \exp \left(\int_{v}^{s} \frac{\partial F_{1}(\zeta(t))}{\partial x} \mathrm{~d} t\right) \frac{\partial F_{1}(\zeta(s))}{\partial y} \exp \left(\int_{0}^{v} \frac{\partial F_{2}(\zeta(t))}{\partial y} \mathrm{~d} t\right) \mathrm{d} v\right] \\
= & \int_{0}^{\tau_{0}} \exp \left(\int_{s}^{\tau_{0}} \frac{\partial F_{2}(\zeta(t))}{\partial y} \mathrm{~d} t\right)\left(-\frac{2 r}{K}+\frac{2 \alpha y_{p}(s)}{\left(c+b y_{p}(s)\right)^{2}}\right) \exp \left(\int_{0}^{s} \frac{\partial F_{2}(\zeta(t))}{\partial y} \mathrm{~d} t\right) \mathrm{d} s \\
& -\int_{0}^{\tau_{0}}\left[\exp \left(\int_{s}^{\tau_{0}} \frac{\partial F_{2}(\zeta(t))}{\partial y} \mathrm{~d} t\right) \frac{b \alpha y_{p}(s)}{\left(c+b y_{p}(s)\right)^{3}} \cdot \int_{0}^{s} \exp \left(\int_{v}^{s} \frac{\partial F_{1}(\zeta(t))}{\partial x} \mathrm{~d} t\right) \frac{\alpha y_{p}(v)}{c+b y_{p}(v)} \exp \left(\int_{0}^{v} \frac{\partial F_{2}(\zeta(t))}{\partial y} \mathrm{~d} t\right) \mathrm{d} v\right] \mathrm{d} s
\end{aligned}
$$

$$
\begin{aligned}
\frac{\partial^{2} \Phi_{2}\left(\tau_{0}, X_{0}\right)}{\partial \tau \partial y} & =\frac{\partial F_{2}\left(\zeta\left(\tau_{0}\right)\right)}{\partial y} \exp \left(\int_{0}^{\tau_{0}} \frac{\partial F_{2}(\zeta(t))}{\partial y} \mathrm{~d} t\right) \\
& =\left(r-\frac{\alpha y_{p}\left(\tau_{0}\right)}{c+b y_{p}\left(\tau_{0}\right)}\right) \exp \left(\int_{0}^{\tau_{0}}\left(r-\frac{\alpha y_{p}(t)}{c+b y_{p}(t)}\right) \mathrm{d} t\right) .
\end{aligned}
$$

Therefore, we can deduce that 


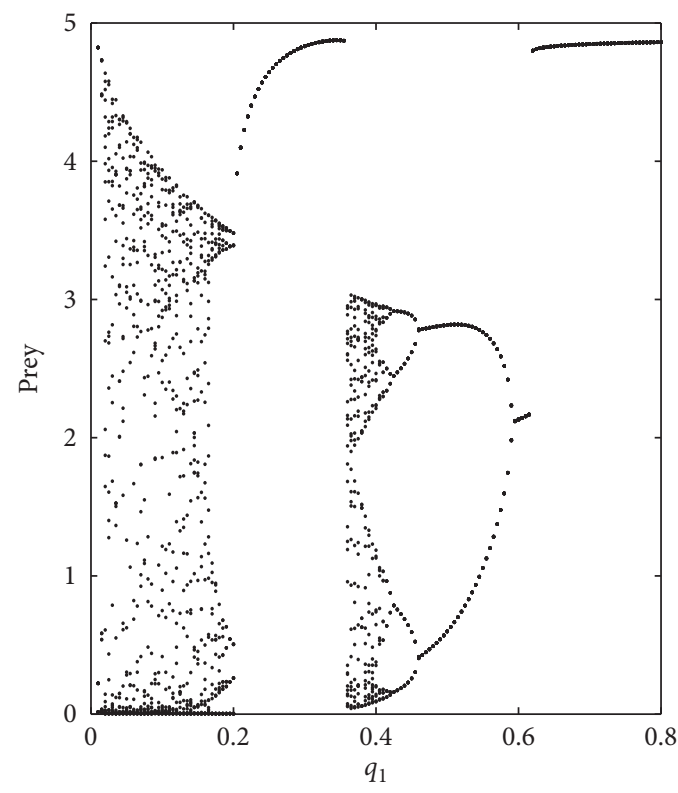

(a)

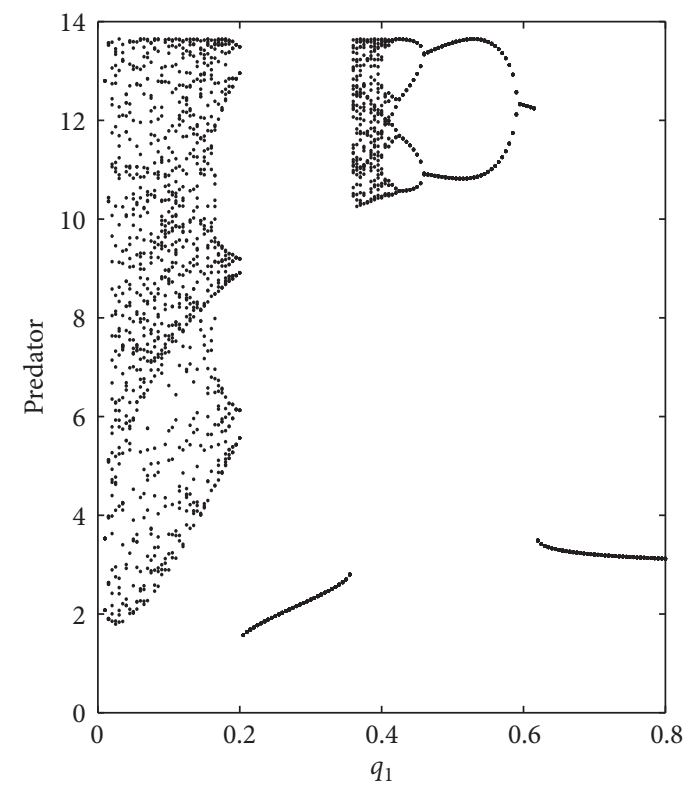

(b)

Figure 6: Bifurcation diagrams of model (1) with respect to $q_{2}$. Parameter values are as follows: $r=2.18, K=5.5, c=1.15, b=0.01, \alpha=$ $0.55, \beta=0.87, \delta=0.34, \theta_{1}=1.2, \theta_{2}=2, q_{2}=0.98, \lambda_{1}=1.4, \lambda_{2}=2.2$, and $T=16.5$.

$$
\begin{aligned}
B= & -\frac{\partial^{2} \Theta_{2}}{\partial x \partial y}\left(\frac{\partial \Phi_{1}\left(\tau_{0}, X_{0}\right)}{\partial \tau}+\frac{\partial \Phi_{1}\left(\tau_{0}, X_{0}\right)}{\partial x} \frac{1}{a_{0}^{\prime}} \frac{\partial \Theta_{1}}{\partial x} \frac{\partial \Phi_{1}\left(\tau_{0}, X_{0}\right)}{\partial \tau}\right) \frac{\partial \Phi_{2}\left(\tau_{0}, X_{0}\right)}{\partial y} \\
& -\frac{\partial \Theta_{2}}{\partial y}\left(\frac{\partial^{2} \Phi_{2}\left(\tau_{0}, X_{0}\right)}{\partial x \partial y} \frac{1}{a_{0}^{\prime}} \frac{\partial \Theta_{1}}{\partial x} \frac{\partial \Phi_{1}\left(\tau_{0}, X_{0}\right)}{\partial \tau}+\frac{\partial^{2} \Phi_{2}\left(\tau_{0}, X_{0}\right)}{\partial \tau \partial y}\right) \\
= & -q_{1}\left(\frac{\partial^{2} \Phi_{2}\left(\tau_{0}, X_{0}\right)}{\partial x \partial y} \frac{1}{a_{0}^{\prime}}\left(q_{2}-\frac{\theta_{2} \lambda_{2}}{\left(1+\theta_{2} y_{p}\left(\tau_{0}\right)\right)^{2}}\right) y_{p}^{\prime}\left(\tau_{0}\right)+\left(r-\frac{\alpha y_{p}\left(\tau_{0}\right)}{c+b y_{p}\left(\tau_{0}\right)}\right) \exp \left(\int_{0}^{\tau_{0}}\left(r-\frac{\alpha y_{p}(t)}{c+b y_{p}(t)}\right) \mathrm{d} t\right)\right), \\
C= & -2 \frac{\partial^{2} \Theta_{2}}{\partial x \partial y}\left(-\frac{b_{0}^{\prime}}{a_{0}^{\prime}} \frac{\partial \Phi_{1}\left(\tau_{0}, X_{0}\right)}{\partial x}+\frac{\partial \Phi_{1}\left(\tau_{0}, X_{0}\right)}{\partial y}\right) \frac{\partial \Phi_{2}\left(\tau_{0}, X_{0}\right)}{\partial y} \\
& -\frac{\partial^{2} \Theta_{2}}{\partial y^{2}}\left(\frac{\partial \Phi_{2}\left(\tau_{0}, X_{0}\right)}{\partial y}\right)^{2}+2 \frac{a_{0}^{\prime}}{b_{0}^{\prime}} \frac{\partial \Theta_{2}}{\partial y} \frac{\partial^{2} \Phi_{2}}{\partial x \partial y}-\frac{\partial \Theta_{2}}{\partial y} \frac{\partial^{2} \Phi_{2}\left(\tau_{0}, X_{0}\right)}{\partial y^{2}} \\
= & 2 q_{1} \frac{b_{0}^{\prime}}{a_{0}^{\prime}} \frac{\partial^{2} \Phi_{2}\left(\tau_{0}, X_{0}\right)}{\partial x \partial y}-q_{1} \frac{\partial^{2} \Phi_{2}\left(\tau_{0}, X_{0}\right)}{\partial y^{2}} .
\end{aligned}
$$

Now, we determine the sign of $B$, For this, let $g(t)=r-\left(\left(\alpha y_{p}(t)\right) /\left(c+b y_{p}(t)\right)\right)$, then $g^{\prime}(t)=(\alpha \delta c y$ $\left.* \exp (-\delta t) /\left(c+b y_{p}(t)\right)^{2}\right)>0$, so $g(t)$ is strictly increasing. Since $\int_{0}^{\tau_{0}} g(t) \mathrm{d} t=\ln \left(1 / q_{1}\right)>0$, which indicates that $g\left(\tau_{0}\right)>0$, i.e. $\left(\partial^{2} \Phi_{2}\left(\tau_{0}, X_{0}\right) / \partial y \partial \tau\right)>0$. Therefore, $B<0$. Defining $\varphi(t)=-(r / K)+\left(\alpha t /(c+b t)^{2}\right)$, it is easy to know that $-(r / K)+\left(\alpha t /(c+b t)^{2}\right) \leq 0$ if $K \alpha<4 r b c$, which means $\varphi\left(y_{p}(t)\right)=-(r / K)+\left(\alpha y_{p}(t) /\left(c+b y_{p}(t)\right)^{2}\right) \leq 0$ for all $y_{p}(t)>0$. So, we have $\left(\partial^{2} \Phi_{2}\left(\tau_{0}, X_{0}\right) / \partial y^{2}\right)<0$. From above, we have $C>0$.

Hence, $\mathrm{BC}<0$; according to Theorem 2 of [33], a supercritical bifurcation occurs at $T=T^{*}$.

\section{Numerical Simulation}

To confirm our theoretical results and facilitate their interpretation, we will focus on the complex dynamics by bifurcation analysis numerically, which can obtain the properties of a dynamics system.

Firstly, we investigate the effect of pulse period $T$ on dynamical of system. Figure 1 shows that model (1) could exist with complex and interesting dynamic behavior with increase of parameter $T$, such as period-doubling bifurcation, period-halving bifurcations, chaos band, and nonunique dynamics, i.e., several attractors may coexist with the 
same $T$. For example, Figure 2 indicates that two different attractors can coexist with each other with the same $T=7$. If we choose different initial value $\left(x_{0}, y_{0}\right)=(1.4,2.1)$ and $\left(x_{0}, y_{0}\right)=(1.8,2.1)$, a $3 T$-periodic solution coexists with $T$ periodic solution, which indicates that the final stable states of pest and natural enemy population depend on their initial densities. All these results confirm that varying impulsive period $T$ could dramatically change the dynamics of model (1).

It follows from Figure 3 that the nonlinear impulsive parameter $\lambda_{1}$ affects the dynamics of model (1). As parameter $\lambda_{1}$ increases, system (1) experiences period-doubling bifurcation, chaotic, period-halving bifurcations, and multiple stability. When $\lambda_{1}=9.4$, two attractors with different amplitudes appear, i.e., a $T$-periodic solution and $2 T$-periodic solution coexist (see Figure 4). Therefore, the initial values of both the pest and predator populations are crucial. The above results reveal that the parameter $\lambda_{1}$ can dramatically change dynamics of system (1).

From the bifurcation diagrams Figure 5, we observe that a positive periodic solution appears when the pest-eradication solution loses its stability. The behavior of positive periodic solution is kept until $q_{2} \approx 3.9$, and then a perioddoubling bifurcation occurs, which means that a $T$-periodic solution disappears suddenly at this point and $2 T$-periodic solution appears. With the increasing of $q_{2}$, a series of period-doubling bifurcations lead model (1) from periodicity to chaos. When $q_{2} \geq 5.16$ and nearby 5.16 , the chaos disappears and a $3 T$-periodic solution appears. As $q_{2}$ increases further, the evidence for $3 T$-periodic solution leading to chaos can be seen. Following these, the system displays a series of period-halving bifurcations.

Similarly, we investigate the effect of parameter $q_{1}$ on dynamic of system. The bifurcation diagrams with respect to parameter $q_{1}$ in the range $[0,0.8]$ are shown in Figure 6 . We can observe that model (1) also displays very complex dynamical behaviors with $q_{1}$ increasing.

\section{Conclusion}

Based on resource limitation, the optimal pest control strategy is that the instantaneous releasing numbers of natural enemies should be adjusted according to the densities of both pest and natural enemy in the field. A more natural understanding is that when the higher the number of pest population or the lower the number of predator population in the field, the higher the number of predator population should be released and the converse is also true. For this, we have investigated effects of nonlinear impulsive perturbations on a predator-prey model with Beddington-DeAngelis functional response. We have proven that there is a global stability of pest-eradication periodic solution if the impulsive period $T<T^{*}$ by using the Floquet theorem and small amplitude perturbation skills, and model (1) is permanent when the period $T>T^{*}$. Hence, $T=T^{*}$ plays a bifurcation threshold, and the system bifurcates to a positive periodic solution via supercritical bifurcation once a threshold condition is reached. By bifurcation diagrams, we can show that the system contains very rich dynamical behavior, including period-doubling bifurcation, periodhalving bifurcations, chaos, and nonunique attractors, i.e., the system could exist with two stable positive periodic solutions and even more complex dynamics (see Figures 2 and 4). Also, bifurcation analyses reveal that the final dynamics of the system depends on the initial densities, and the nonlinear impulsive may result in complexity of pest control. All those results confirm that the pest control strategy should be carefully designed once the nonlinear impulsive control measures have been taken into account.

Based on the present study, we found that the system with nonlinear impulsive control actions provides more rich results and more realistic than the previous systems with linear impulsive control, and thus nonlinear impulsive control should be taken into account when implementing integrated pest management. However, the aim of IPM should reduce pest populations to below the economic threshold rather than eradication, which can be naturally and accurately described by the state-dependent impulsive differential equations and result in more difficulty for analyzing the global dynamics. We leave for future research.

\section{Data Availability}

There were no data used to support this study.

\section{Conflicts of Interest}

The authors declare that they have no conflicts of interest.

\section{Acknowledgments}

This work was supported by the National Natural Science Foundation of China (NSFC, no. 61772017), the Natural Science Basic Research Plan in Shaanxi Province of China (no. 2020JM-569), the Shaanxi Province Department of Education Fund (no. 18JK0393), and the Project of Improving Public Scientific Quality in Shaanxi Province (no. 2020PSL(Y)073).

\section{References}

[1] R. E. Stinner, "Efficacy of inundative releases," Annual Review of Entomology, vol. 22, no. 1, pp. 515-531, 1977.

[2] J. L. Capinera and D. R. Dent, "Integrated pest management," The Florida Entomologist, vol. 80, no. 2, pp. 307-308, 1997.

[3] J. C. Van Lenteren and J. Woets, "Biological and integrated pest control in greenhouses," Annual Review of Entomology, vol. 33, no. 1, pp. 239-269, 1988.

[4] H. I. Freedman, "Graphical stability, enrichment, and pest control by a natural enemy," Mathematical Biosciences, vol. 31, no. 3-4, pp. 207-225, 1976.

[5] Y. N. Xiao and F. Van Den Bosch, "The dynamics of an ecoepidemic model with biological control," Ecological Modelling, vol. 168, no. 1-2, pp. 203-214, 2003.

[6] W. W. Murdoch, J. Chesson, and P. L. Chesson, "Biological control in theory and practice," The American Naturalist, vol. 125, no. 3, pp. 344-366, 1985.

[7] S. Nundloll, L. Mailleret, and F. Grognard, "Influence of intrapredatory interferences on impulsive biological control 
efficiency," Bulletin of Mathematical Biology, vol. 72, no. 8, pp. 2113-2138, 2010.

[8] S. Nundloll, L. Mailleret, and F. Grognard, "Two models of interfering predators in impulsive biological control," Journal of Biological Dynamics, vol. 4, no. 1, pp. 102-114, 2010.

[9] N. Bajeux, F. Grognard, and L. Mailleret, "Augmentative biocontrol when natural enemies are subject to Allee effects," Journal of Mathematical Biology, vol. 74, no. 7, pp. 1561-1587, 2017.

[10] V. Lakshmikantham, D. D. Bainov, and P. S. Simeonov, Theory of Impulsive Differential Equations, World Scientific, Singapore, 1989.

[11] D. D. Bainov and P. S. Simeonov, "Impulsive differential equations: periodic solutions and applications,"PitmanMonographs and Surveys in Pure and Applied Math, vol. 66,Longman Scientific and Technical, New York, NY, USA, 1993..

[12] X. Liu and L. Chen, "Global dynamics of the periodic logistic system with periodic impulsive perturbations," Journal of Mathematical Analysis and Applications, vol. 289, no. 1, pp. 279-291, 2004.

[13] B. Liu, Y. J. Zhang, L. S. Chen, and L. H. Sun, "The dynamics of a prey-dependent consumption model concerning integrated pest management," Acta Mathematica Sinica, English Series, vol. 21, no. 3, pp. 541-554, 2005.

[14] B. Liu, Y. Zhang, and L. Chen, "Dynamic complexities in a lotka-volterra predator-prey model concerning impulsive control strategy," International Journal of Bifurcation and Chaos, vol. 15, no. 02, pp. 517-531, 2005.

[15] S. Tang and R. A. Cheke, "State-dependent impulsive models of integrated pest management (IPM) strategies and their dynamic consequences," Journal of Mathematical Biology, vol. 50, no. 3, pp. 257-292, 2005.

[16] S. Tang and R. A. Cheke, "Models for integrated pest control and their biological implications," Mathematical Biosciences, vol. 215, no. 1, pp. 115-125, 2008.

[17] S. Tang, Y. Xiao, and R. A. Cheke, "Multiple attractors of hostparasitoid models with integrated pest management strategies: eradication, persistence and outbreak," Theoretical Population Biology, vol. 73, no. 2, pp. 181-197, 2008.

[18] Q. Xiao and B. Dai, "Periodic solutions generated by impulses for state-dependent impulsive differential equation," Discrete Dynamics in Nature and Society, vol. 2015, Article ID 816325, 7 pages, 2015.

[19] Z. Xiang, S. Tang, C. Xiang, and J. Wu, "On impulsive pest control using integrated intervention strategies," Applied Mathematics and Computation, vol. 269, pp. 930-946, 2015.

[20] S. Tang, W. Pang, R. A. Cheke, and J. Wu, "Global dynamics of a state-dependent feedback control system," Advances in Difference Equations, vol. 2015, no. 322, 2015.

[21] C. T. Li and S. Y. Tang, "The effects of timing of pulse spraying and releasing predator and releasing periods on dynamics of generalized predator-prey moedl," International Jouranla of Biomathematics, vol. 5, no. 1, pp. 1-28, 2012.

[22] W. J. Qin, S. Y. Tang, and R. A. Cheke, "The effects of resource limitation on a predator-prey model with control measures as nonlinear pulses," Mathematical Problems in Engineering, vol. 2014, Article ID 450935, 13 pages, 2014.

[23] G. Tang, S. Tang, and R. A. Cheke, "Global analysis of a holling type II predator-prey model with a constant prey refuge," Nonlinear Dynamics, vol. 76, no. 1, pp. 635-647, 2014.

[24] S. Y. Tang, B. Tang, A. L. Wang, and Y. N. Xiao, "Holling II predator-prey impulsive semi-dynamic model with complex poincare map,” Nonlinear Dynamics, vol. 81, pp. 1579-1596, 2015.

[25] L. Dong and L. Chen, "A periodic predator-prey-chain system with impulsive perturbation," Journal of Computational and Applied Mathematics, vol. 223, no. 2, pp. 578-584, 2009.

[26] H. Zhang, P. Georgescu, and L. Chen, "On the impulsive controllability and bifurcation of a predator-pest model of IPM," Biosystems, vol. 93, no. 3, pp. 151-171, 2008.

[27] H. K. Baek, "Qualitative analysis of Beddington-DeAngelis type impulsive predator-prey models," Nonlinear Analysis: Real World Applications, vol. 11, no. 3, pp. 1312-1322, 2010.

[28] S. L. Sun and G. H. Guo, "Dynamics of a beddingtondeangelis type predator-prey model with impulsive effect," Journal of Mathematics, vol. 2013, Article ID 826857, 11 pages, 2013.

[29] C. T. Li and S. Y. Tang, "Complex dynamics and coexistence of period-doubling and period-halving bifurcations in an integrated pest management model with nonlinear impulsive control," Advances in Difference Equations, vol. 514, pp. 1-23, 2020.

[30] I. U. Khan and S. Y. Tang, "The impulsive model with pest density and its change rate dependent feedback control," Discrete Dynamics in Nature and Society, vol. 2020, Article ID 1561241, 20 pages, 2020.

[31] X. Wang, Y. Tian, and S. Y. Tang, "A holling type II pest and natural enemy model with density dependent IPM strategy," Mathematical Problems in Engineering, vol. 2017, Article ID 8683027, 12 pages, 2017.

[32] C. Li and S. Tang, "Analyzing a generalized pest-natural enemy model with nonlinear impulsive control," Open Mathematics, vol. 16, no. 1, pp. 1390-1411, 2018.

[33] A. Lakmeche and O. Arino, "Bifurcation of non-trivial periodic solutions of impulsive differential equations arising chemotherapeutic treatment," Dynamics of Continuous, Discrete and Impulsive System, vol. 7, no. 2, pp. 265-287, 2000. 